\title{
ACCOUNT OF A PATIENT
}

\section{UPON WHOM \\ OVARIOTOMY WAS PERFORMED TWICE;}

\author{
WITH REMARKS.
}

BY

T. SPENCER WELLS, F.R.C.S., SURGEON TO THE SAMARITAN HOSPITAI, LECTURER ON SURGERY, ETC.

Received April 20th.-Read June 9th, 1868.

IN November, 1862, I was consulted by a married woman, forty-two years of age, from whom an ovarian tumour had been removed six months before by another surgeon. She left the institution in which ovariotomy was performed three weeks after the operation; but about a week after going home she became sick, and noticed an enlargement on the right side of the abdomen. She consulted Sir Charles Locock, who had seen her before the first operation, and who told her that another tumour was growing. Sir Charles saw her again in October, told her that the tumour was increasing, and advised her to wait about three months before having a second operation performed.

When she came to me I was not aware that ovariotomy had ever been performed twice on the same patient. A case had been recorded in America where one surgeon had attempted to remove an ovarian tumour, but failed in his attempt, and another surgeon had afterwards succeeded. 
But I could find no case on record in which a patient liad recovered after ovariotomy, and had afterwards undergone the operation a second time on account of disease of the remaining ovary. I was, therefore, very anxious to obtain the opinion of eminent men respecting this patient, and I believe that several who saw her with me looked upon the case as unprecedented. But I have since learned that Dr. Attlee, of Philadelphia, has performed ovariotomy successfully upon a patient from whom Dr. Clay, of Manchester, had removed an ovarian tumour of the opposite side sixteen years before. The case which I bring before the Society is, therefore, not the first in which ovariotomy has been performed twice on the same patient; but it still appears to be sufficiently interesting to justify me in bringing it thus prominently before the profession.

When the patient first consulted me the tumour filled the greater part of the abdomen below the level of the umbilicus. On the right side it was elastic and obscurely fluctuating, while on the left side it was very hard. The uterus seemed to be closely connected with the hard tumour on the left side. The catamenia had not appeared since the first operation; but at every monthly period she had had pains in the back and thighs, lasting for a day, and leaving pain in the right hip and swelling of the breasts for two or three days. Ever since the operation she had complained of pains below the epigastrium, with flatulence, and the bowels never acted without purgative medicine.

On the 25th of December, the usual symptoms returned with the monthly period; but this time the discharge came on, not excessive in amount, without clots, and lasted five days.

On the 3rd of January, 1863, the girth of the abdomen at the umbilical level was thirty-five inches, and forty inches over the most prominent portion of the abdomen, which was about three inches below the umbilicus. The distance from symphisis pubis to umbilicus was eleven inches, and from umbilicus to ensiform cartilage six inches. From one anterior superior spinous process of the ilium across the 
abdomen to the opposite process the distance was eighteen inches. There was a hard cicatrix three-quarters of an inch to the right of the linea alba, extending from two inches below the umbilicus to seven inches from this point; the cicatrix thus being five inches long. The tumour moved freely beneath the abdominal wall, but there was a slight. crepitus felt nearly all over it as it moved. There was still the same extreme hardness of that portion of the tumour to the left of the umbilicus, and the same elasticity and obscure fluctuation of the portion to the right, as at my first examination.

It was evident that the connection between the uterus and the tumour was close, for as the patient lay on her side the uterus was pulled almost out of reach. The uterine sound passed to four and a half inches; not towards the hard tumour on the left side, but towards the right side, its point being distinctly perceptible just above the right internal abdominal ring. Fluctuation could be detected (though not very distinctly) in the vagina, below the hard portion of the tumour on the left side.

I communicated with Sir Charles Locock upon all these points, and proposed to make an exploratory incision, and to be guided by the connection of the tumour as to further proceedings. Sir Charles approved of this suggestion, and added, "the operation affords the only hope of relief."

Before proceeding to operate, I considered whether it would be better to make the incision through the linea albathat is, within an inch of the cicatrix-or in one of the lineæ semilunares. But as there was some doubt whether the tumour was a growth from the right ovary, or a growth of some portion which had not been removed from the left side-in other words, whether the uterus was pulled or pushed to the right side-it appeared to be safer to cut in the median line than to run any risk of making the incision on the side opposite to the uterine attachment.

I performed the operation on the 13th of January, 1863. Mr. Clover administered chloroform, and I was ably assisted by Dr. Savage, Dr. Drage, of Hatfield, and Mr. Webb, of 
Welwyn. I made an incision over the linea alba, threequarters of an inch to the left of the cicatrix, and parallel with the lower four inches of it. On dividing the peritoneum, the tumour was seen to be composed of very thinwalled cysts, very tensely distended with clear fluid. These cysts, or rather divisions of a multilocular cyst, passed successively through the opening in the abdominal wall as $\mathrm{Dr}$. Savage pressed the tumour from behind forwards. Several filmy layers of organized lymph and a layer of expanded omentum were pressed outwards before the cyst, and were divided on a director. A piece of omentum which adhered both to the cyst and to the abdominal wall near the upper part of the incision was easily separated, and the tumour was then pressed out entire, without emptying any of the cysts. The pedicle was short, but it was easily secured by a clamp. It passed in the usual manner from the right side of the uterus. The uterus seemed to be of natural size. No remnant of the left ovary was found. After cutting away the tumour, there was some oozing of blood around the clamp, but it was stopped by tying a ligature tightly round the pedicle beneath the clamp. One bleeding vessel in the abdominal wall, and two in the omentum, were also tied. Just above the upper angle of the wound a long coil of small intestine adhered firmly to the abdominal wall. As the patient had complained of pain at this spot, and had suffered from constipation ever since the first operation, I examined the connection between the intestine and the abdominal wall to see if they could be separated safely; but the adhesions appeared to be so very close that I did not attempt to effect any separation. The wound was closed by deep and superficial silk sutures.

The cyst is placed on the table of the Society. It is a good specimen of what is known as the compound proliferous cyst ; and it is curious that the small groups of minute cysts not only grow into the cavity of the parent cyst, or project inwards, but also perforate the cyst-wall and project into the peritoneal cavity.

The patient rallied remarkably well after the operation, 
and for forty-eight hours seemed to be recovering. Two small opiates were given on account of pain, but reaction was not excessive. The aspect was good; and the tongue, though white, was moist. The pulse was about 100. I removed the clamp forty-four hours after operation, as it seemed to be lying quite loose on the wound; the ligature which had been tied beneath it also came away with a shred of dead fibrous tissue. There was no bleeding. I also removed three of the sutures.

On the 16th, the third day after operation, there was some flatulent distension of the abdomen, and frequent eructation, but no vomiting. The rectum was cleared by an enema. At 9 p.m., during one of the " fits of belching," as the nurse called them, the lower part of the wound gave way, and a knuckle of intestine protruded. A good deal of fetid serum also escaped. I returned the intestine, reapplied three sutures deeply, and the patient did not seem to be worse.

On the next day, the 17 th, there was free fetid discharge from the lower part of the wound, and vomiting became troublesome; but the pulse was not more than 110, and the aspect was good.

On the 18th, the pulse had risen to 120 , but the tongue was moist and cleaning from the edges, and the colour of cheeks and lips very good. Still she was decidedly weaker, and the tympanites was increasing.

She continued to become weaker all the next day, notwithstanding the free use of stimulants and nourishment both by the mouth and the rectum; and she died on the seventh day, or 154 hours after the operation.

Decomposition of the body took place very rapidly. There was a good deal of fetid serum in the peritoneal cavity, and some traces of recent peritonitis were also shown by flakes of lymph. There was no blood nor clot to be seen, and only one or two shreds of sloughy tissue at the spot where the tumour had been removed from the right side of the uterus. The peduncle of the tumour first removed connected the left side of the uterus closely with the abdominal wall. 
The adhering portion of intestine observed during my operation was so closely attached to the abdominal wall that it was difficult to separate it by dissection; and the greater part of the omentum also adhered to the abdominal wall.

This case alone is sufficient to prove that ovariotomy may be performed twice on the same patient without any unusual difficulty. What the risk may be as compared with the risk of first operations can only be ascertained by a number of cases.

Reflection upon this case would seem to suggest that, in performing the operation for the second time on the same patient, it may prove advisable to make the incision at some distance from the cicatrix left after the first operation; or, if the incision be made near the cicatrix, it may be necessary to leave the sutures longer than in ordinary cases, as the process of union may be slower near a cicatrix than in an uninjured part.

The lessons suggested to those who perform ovariotomy under ordinary circumstances are--

1. That the operator should be careful not only to remove every portion of an ovarian tumour on one side, if it be possible to do so, but also to examine the opposite ovary carefully, and to be guided in his practice by the knowledge that if the ovary be not healthy and be left behind, morbid growth will probably take place, and a second operation be required.

2. That in uniting the wound in the abdominal wall the divided edges of peritoneum should be brought closely together in the manner which I was the first to propose in a paper presented to this Society five years ago. The adhesions between the omentum and intestine and the abdominal wall observed in this patient precisely resemble the condition which I have observed in dogs, rabbits, and guinea pigs after opening the abdomen, and closing the wound by sutures which have not included the peritoneum. In every case, the serous bag was completed bv adhesion of portions of omentum or intestine, or of both; and in some cases the 
animals were greatly inconvenienced by these attachments. But in all the cases where two surfaces of peritoneum had been pressed together by the sutures, union took place without any adhesion of intestine or omentum. Several preparations are placed on the table of the Society which illustrate this fact, and show that the supposed danger of the sutures coming into contact with and irritating the viscera, or of the tracks of the sutures forming fistulous openings between the skin and the peritoneal cavity, are purely imaginary dangers. It is demonstrable that the folding together of the peritoneal borders of the wound completely conceals or shuts off the sutures from the cavity of the peritoneum ; and even if the sutures are left long enough to form sinuses, these must still be external to the peritoneal cavity.

The surgeon who performed the first operation on this patient does not include the peritoneum in his sutures; and I think that the adhesion of intestine and omentum with the consequent discomfort and constipation suffered by the patient-evils observed in animals so treated, but never observed in those where the peritoneum had been included in the sutures, nor in any of the patients who have either died or recovered under my care-are strong arguments in favour of that mode of uniting all penetrating wounds of the abdominal wall which I have submitted to the consideration of the Profession in this and former papers brought before the Society. 